\title{
Los Elementos y Dinámicas de la Evaluación en el Área de matemáticas, Grado Quinto
}

José Albeiro Zambrano Castañeda* Gerardo Toca Ramírez **

Artículo de reflexión

Fecha de Recepción: 15 octubre 2017.

Fecha de Aprobación: 25 febrero 2018.

\section{Resumen}

El presente artículo de reflexión emerge de un ejercicio de deliberación en torno a los elementos de evaluación que privilegian cuatro docentes, quienes orientan el área de Matemáticas, de grado quinto, en la Institución Educativa Rancho Grande, del Municipio de Rondón (Boyacá). El análisis cualitativo, llevado a cabo durante el proceso de indagación, se orientó hacia la necesidad que los docentes tienen de fortalecer la concepción sobre los elementos de la evaluación, para hacer buen uso de los resultados de las valoraciones, de tal manera que estas ayuden a mejorar los procesos de aprendizaje de cada uno de los estudiantes. De esta manera, se deduce que la evaluación formativa articulada con la resolución de problemas, proporciona al docente una herramienta que facilita la toma de decisiones orientadas a ofrecer una realimentación continua $y$ oportuna, para establecer acciones de mejoramiento educativo en el aula de clase.

Palabras clave: evaluación formativa, resolución de problemas, matemáticas, elementos de la evaluación, aprendizaje.
* Institución Educativa Jaime Jácome, Boyacá Colombia

albeirozam14@outlook.es

** Institución Educativa Rancho Grande, Boyacá Colombia

licgertora@yahoo.es

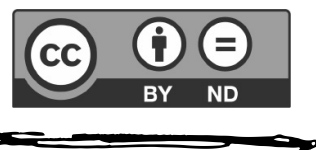




\section{Introducción}

No resulta exagerado decir que, en el campo académico de educación básica primaria existen varias teorías que abordan el tema sobre evaluación, pero realmente muy pocas se enfocan a las dinámicas de didáctica práctica. El objeto de este análisis es determinar, a través de la observación, los elementos que privilegian los docentes en la evaluación, en el área de Matemáticas de los estudiantes, de grado $5^{\circ}$, de la Institución Educativa Rancho Grande, del Municipio de Rondón (Boyacá); para, luego, ofrecer alternativas didáctico pedagógicas, que contribuyan al desarrollo de la competencia Resolución de Problemas, en aras de un fortalecimiento en el proceso de valoración de los aprendizajes.

En este artículo, se pretende dinamizar la evaluación teniendo en cuenta las etapas de la resolución de problemas como insumo para generar nuevos instrumentos de valoración de aprendizajes, reconociendo los diferentes procesos formativos, para que el docente continúe con el enriquecimiento de su propio sistema de evaluación.

\section{Justificación}

Este ejercicio de reflexión se torna interesante toda vez que se puede hablar de una forma de rescatar la evaluación como herramienta diagnóstica y didáctica, que sirva de guía orientadora para el docente y los estudiantes, implicando, de esta manera, un cambio en las dinámicas de clase y una evaluación más formativa que evidencie el aprendizaje del estudiante, no solamente de manera individual sino, a la vez, su desarrollo a partir del trabajo grupal y cooperativo.

\section{Antecedentes}

Algunas tendencias educativas y pedagógicas consideran la evaluación como un proceso técnico de verificación de resultados de aprendizaje, acorde con objetivos predeterminados. Sin embargo, la evaluación escolar es un procedimiento complejo, pues en él confluyen gran variedad de factores, algunos de ellos explícitos y otros menos advertidos y, por ende, se hallan implícitos. Los primeros provienen desde la escuela y están asociados a los contenidos educativos, normas, regulaciones, criterios, procedimientos, exigencias administrativas y condiciones de trabajo en las aulas; factores que no solo orientan la evaluación, sino que, igualmente, la afectan. Los implícitos provienen desde los propios profesores quienes aplican criterios e implementan prácticas docentes relacionadas con sus concepciones respecto de evaluación y aprendizaje, valorando, de una u otra manera, sus fines, sentido y trascendencia y determinando, en gran medida, la naturaleza de sus prácticas de enseñanza y evaluativas e incidiendo, de manera crítica, en los resultados de los procesos formativos.

Se insiste que, el proceso educativo que se realiza en el interior de las aulas, lo conforman distintas 
acciones. La evaluación es parte primordial de este proceso y está presente durante y desde el momento mismo de la planeación; la evaluación que se realiza en el aula es empleada no solo con el objetivo de mejorar el proceso de enseñanza y aprendizaje, sino, también, para justificar la clasificación o exclusión de los estudiantes de la Institución Educativa.

De acuerdo con esta premisa, en esta labor reflexiva se determina las concepciones de evaluación educativa que subyacen en las prácticas de los docentes al momento de validar los aprendizajes de los estudiantes, la importancia de los resultados de la evaluación y el grado de responsabilidad de cada uno de los actores involucrados en el proceso de evaluación, teniendo en cuenta que en la educación tradicional es muy común encontrar el proceso enseñanza-aprendizaje-evaluación como una unidad y no por fases.

\section{Método}

Este trabajo indaga, determina y caracteriza, en la institución educativa escenario de consulta, las prácticas referente a: - los tipos de evaluación, - los momentos en que los estudiantes son evaluados, - los diferentes procesos objeto de evaluación; - si se está evaluando por niveles de competencias, ritmos de procesos o actitudes; - los principios evaluativos que se están aplicando, - la clase de evaluación que se está realizando (ya sea diagnóstica o formativa) - o si solo se está constatando la obtención de lo propuesto inicialmente; además, si están de acuerdo con "las nuevas apuestas en este campo del conocimiento que proponen que la evaluación educativa, en los niveles de enseñanza básica y media, tengan única y exclusivamente propósitos formativos, es decir, de aprendizaje para todos los sujetos que intervienen en ella". (Ministerio de Educación Nacional , 2009, pág. 22)

Una vez, organizados los datos recabados con los instrumentos utilizados (entrevista, revisión documental), se procedió a triangular o correlacionar dicha información para determinar la coherencia entre las prácticas evaluativas y el sistema institucional de evaluación de estudiantes. Posteriormente, se muestran los hallazgos obtenidos, y se destacan los elementos que aportan al ámbito de la evaluación educativa.

Revisando este panorama, teniendo en cuenta los instrumentos aplicados, los registros escritos y grabaciones, se analiza la información que permita formular el interrogante reflexivo que, una vez, resuelto sirva de soporte para concluir lo planteado: ¿Qué elementos para el diseño de evaluación en el área de Matemáticas, del grado $5^{\circ}$, emergen del análisis de las prácticas pedagógicas de los docentes de la Institución Educativa Rancho Grande, del Municipio de Rondón, Boyacá?

Con base en lo anterior y con el interés de apoyar los procesos educativos y contribuir, a la vez, a transformar las prácticas pedagógicas de los docentes, durante el desarrollo de esta iniciativa, 
se presentaron varias fases. La primera consistió en hacer un diagnóstico, en el establecimiento educativo citado, por medio del reconocimiento de las necesidades donde se realizaron registros de las posturas respecto a la evaluación, las formas cómo evalúan dentro del contexto de aula. La segunda consistió en el uso de la heteroevaluación, la coevaluación y la autoevaluación, como referentes para establecer diferentes elementos evaluativos usados por los docentes en los diferentes momentos de la clase. La tercera fase consistió en plantear elementos de evaluación en las prácticas de aula, con el fin de desarrollar la competencias efectivas del área de Matemáticas de grado $5^{\circ}$, estrategia pedagógica que ayude a los docentes a mejorar los procesos de aprendizaje de los estudiantes en la clase.

\section{Soportes y acciones}

En este perfil reflexivo, enfocado a lo cualitativo, de naturaleza descriptiva, se aborda a Gómez y Roquet (2012), quien ilustra sobre el proceso de transformar la realidad educativa $y$ social, orientando su acción hacia la resolución de problemas partiendo desde lo reflexivo hasta lo transformador. Con base en lo anterior, este análisis se sustenta en un estudio diagnóstico donde se emiten juicios sobre los procesos evaluativos actuales en la institución educativa. Igualmente, se acude a documentales en razón a que se acude a la consulta de registros e informes sobre los resultados de los procesos de enseñanza-aprendizaje llevados a cabo en el aula de clase y de campo, con entrevistas y encuestas a los docentes de matemáticas de la Institución Educativa Rancho Grande del Municipio de Rondón (Boyacá); la tarea consiste en determinar los elementos que privilegian en la evaluación, del área de Matemáticas para desarrollar las competencias efectivas en los estudiantes, a partir de alternativas pedagógicas que fortalezcan el proceso de valoración de los aprendizajes. La muestra seleccionada está conformada por cuatro docentes de primaria que orientan la formación en el grado $5^{\circ}$. Los educadores aportaron la información requerida mediante las respuestas en la entrevista semiestructurada, encuestas y los registros de observación del grupo en estudio, (Salkind, 1999).

No sobra mencionar que, una vez se obtuvo la recolección de información, se procede al análisis pertinente, teniendo como norte el objetivo propuesto en esta reflexión. Así mismo, se hizo una revisión del PEI (Proyecto Educativo Institucional) con el propósito de identificar si las prácticas evaluativas aplicadas por los docentes son establecidas en el marco de políticas institucionales como lo es el SIEE (Sistema Institucional de Evaluación de Estudiantes) y si estas buscan dar cuenta del proceso individual y de la formación integral.

\section{Resultados y discusión}

En el desarrollo de este análisis, se puede afirmar que la mayoría de las prácticas evaluativas solamente se utilizan para emitir una calificación o para 
promover un estudiante de un grado a otro; inclusive, los planes de apoyo se convierten en talleres adicionales que no contribuyen en procesos de aprendizaje de los estudiantes, pues sin orientación del docente es complejo desarrollar las actividades propuestas, por ello, muchas veces los niños y niñas presentan apatía para ir a la escuela.

Sobre los elementos que privilegian los docentes en la evaluación para área de matemáticas, en la Institución Educativa Rancho Grande, del Municipio de Rondón Boyacá, los profesores del área opinan en general que, en la formación educativa se evidencia que es de menor importancia la evaluación valorativa o sumativa, frente a la formativa, aspecto que en la realidad no se aprecia, teniendo en cuenta que los alumnos reciben evaluaciones calificadas y no cualificadas, es decir, el juicio de valor es numérico y algunas veces incluye solo comentarios cortos sobre la necesidad de aumentar el esfuerzo o mejoramiento en el aprendizaje.

Al respecto, Aldana \& Wagner (2012) proponen un modelo pedagógico que articule cambios en las prácticas de enseñanza y de aprendizaje e incluyen cambios en la organización del espacio, los tiempos y los tipos de relación entre los estudiantes, los profesores y la administración. Se sugiere que los profesores deben ser organizados en torno al logro de los objetivos que pretende el proceso didáctico con sus estudiantes, deben recoger los campos conceptuales, en este caso, de las matemáticas, que hacen que el sujeto que aprende dé cuenta de los saberes propios del contexto de las matemáticas que configuran el perfil profesional.

De esta manera, la filosofía al respecto de la institución debe basarse en la nueva cultura evaluativa, que busca resaltar el papel que representa la evaluación en los procesos de enseñanza-aprendizaje.

Con base en lo anterior, al identificar los métodos de evaluación que privilegian los maestros en el área de Matemáticas, en la Institución Educativa Rancho Grande, del Municipio de Rondón Boyacá, se observó que hay docentes que solo basan el logro de resultados, en el mecanismo de los instrumentos que generan cifras cuantitativas para mostrar el nivel de aprendizaje de los educandos, dejando de lado el verdadero potencial formativo que puede aportar una estrategia evaluativa con fondo analítico y organizado, que permita implementar pedagogías que favorezcan el interés de los alumnos por aprender. De esta forma, las herramientas evaluativas no solo serán registros que proporcionan resultados.

En lo relacionado con la innovación en los procesos de evaluación, los docentes de matemáticas en mención manifestaron que conocen los conceptos que integran sus mecanismos con los agentes que la regulan. Sin embargo, no se lleva a cabo una práctica dinámica de lo que se propone, porque una cosa es el discurso sobre la necesidad de cambio y mejoramiento al respecto y otra su aplicación.

Los profesores en cuestión no tienen un claro concepto sobre la destinación y uso que le deben dar a los resultados
Aldana \& Wagner (2012) proponen un modelo pedagógico que articule cambios en las prácticas de enseñanza y de aprendizaje e incluyen cambios en la organización del espacio, los tiempos y los tipos de relación entre los estudiantes, los profesores y la administración. 
Flores \& Gómez (2008) caracterizan la evaluación de los procesos de enseñanza y aprendizaje desde la perspectiva de un modelo de enseñanza centrado en el estudiante, ya que, de acuerdo a ellos, esta necesita tener en cuenta todos los aspectos que se consideran en el modelo de enseñanza, tanto los que corresponden a las competencias como a las cualidades personales.

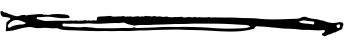

mostrados por sus alumnos, aunque perciben esta situación como un medio de carácter formativo. Sin embargo, algunos docentes promueven el uso de la evaluación diagnóstica en donde cada individuo puede autorregular su aprendizaje y fortalecer su función comprensiva y de autoaprendizaje.

Villa \& Henao (2009), en este aspecto, propone desarrollar el proceso evaluativo con base en el enfoque del pensamiento matemático, lo cual implica el manejo de una pedagogía especial del área, de acuerdo con los procesos aplicados y al conocimiento adquirido que le permita su entorno. Para el desarrollo de las matemáticas y de los planteamientos de la propuesta de evaluación, se proponen métodos que: aproximen el conocimiento a través de situaciones y problemas que propician la reflexión, exploración y apropiación de los conceptos matemáticos; desarrollen el razonamiento lógico y analítico para la interpretación y solución de problemas; estimulen la aptitud matemática con actividades lúdicas que ponen a prueba la creatividad y el ingenio de los estudiantes.

Los docentes, en general, tienen la preocupación constante de que sus estudiantes desarrollen una mejora continua en el aprendizaje, sin importar que para esto deban utilizar momentos diferentes a los horarios escolares, donde muchas veces tienen que intervenir sobre riesgos de vulnerabilidad, que van más allá de su responsabilidad profesional.

Se comprende que, es una constante que no todos los profesores centran el análisis de los resultados del proceso de enseñanza-aprendizaje en la identificación de las verdaderas necesidades cognitivas de los alumnos.

Flores \& Gómez (2008) caracterizan la evaluación de los procesos de enseñanza y aprendizaje desde la perspectiva de un modelo de enseñanza centrado en el estudiante, ya que, de acuerdo a ellos, esta necesita tener en cuenta todos los aspectos que se consideran en el modelo de enseñanza, tanto los que corresponden a las competencias como a las cualidades personales. Estos autores concluyen que, la evaluación del proceso de enseñanza y aprendizaje en el aula, debe estar acorde con la concepción de conocimiento y la metodología de enseñanza que se ponga en práctica. En consecuencia, si se plantea cambiar la educación centrada en el docente a una educación centrada en el estudiante, es preciso cambiar el concepto de evaluación y diseñar los instrumentos adecuados para hacer que tal evaluación sea efectiva.

Ahora bien, se debe entender las concepciones sobre la evaluación del aprendizaje que tienen los docentes de matemática en la Institución Educativa -escena de la consulta reflexiva-. Allí, según el concepto de los mismos profesores, existe en ellos la conciencia de la misión de forjar un aprendizaje claro en sus alumnos, pese a que no se plasma con claridad el resultado obtenido del esfuerzo que ellos realizan, a través de la evaluación, frente a la calidad de los avances mostrados en el aula, de manera que se puedan proponer estrategias para corregir 
los problemas latentes. Moreno \& Ortiz (2016) enfocan la evaluación dentro del proceso de enseñanzaaprendizaje, particularmente en el área de Matemática, analizando las concepciones que, sobre evaluación, poseen los profesores de esta asignatura.

Según los autores, los docentes centran la evaluación para calificar los conocimientos, habilidades, fallas o deficiencias, verificar los logros alcanzados respecto de los objetivos y los contenidos, específicamente de forma cuantitativa, por cuanto consideran que se realiza para colocar una nota, que demuestra la capacidad del alumno y su nivel de aprendizaje, lo cual al fin del proceso permite la verificación del logro de objetivos propuestos en el currículo establecido.

En estas circunstancias se aprecia que, los docentes de matemáticas de la institución, campo de estudio, entienden la necesidad de implementar grupos de trabajo para diseñar formas de evaluación acordes al entorno social, familiar y educativo de sus alumnos, pero también, en la entrevista realizada, resaltan la importancia de adelantar procesos de capacitación para concebir métodos evaluativos en beneficio de los educandos.

\section{Propuestas de estrategias para la evaluación}

El análisis de resultados del trabajo, permite el surgimiento de propuestas en la creación de estrategias, para que la evaluación impacte directamente en las prácticas de aula. Aquí, se reconocen dificultades y fortalezas, elementos que permiten propender por el mejoramiento continuo en procesos de enseñanza y en procesos de aprendizaje. Además, se sugiere que la evaluación debe ser participativa (Cardona, Jaramillo, \& Navarro, 2016), ya que le ayuda al alumno a saber: si está mejorando su aprendizaje, a ubicar su desempeño respecto de los objetivos y en comparación con sus compañeros, a reconocer la ayuda que necesita y lo que debe hacer impulsándolo a asumir la responsabilidad de su rendimiento (autoevaluación). A los padres, se debe permitir observar avances en el desempeño pedagógico de sus hijos y reconocer formas en las que pueden apoyarlos para mejorar. En la escuela, la evaluación de aprendizajes en el aula debe permitir identificar los logros en la implementación del currículo y las necesidades de trabajo cooperativo, para resolver los problemas señalados $y$, de una u otra forma, que contribuyan al mejoramiento de la calidad educativa de la institución.

Vale mencionar que, en este análisis del estudio hecho en la Institución Educativa Rancho Grande del Municipio de Rondón (Boyacá), se parte de la premisa que evaluar es valorar los diferentes procesos que un estudiante ha mostrado y su apropiación en su contexto institucional. Por lo tanto, una buena valoración es fundamental para el desarrollo personal y social del educando, de tal manera que todos los ciudadanos aporten a la sociedad en la construcción de la justicia, la equidad y desarrollo humano a través de sus capacidades. Es evidente que, el control evaluativo requiere ser mejorado para 
que sea un instrumento eficiente, que permita los logros propuestos al inicio del periodo escolar.

Respecto a los estudiantes, es importante generarles espacios donde puedan expresar sus ideas previas al nuevo conocimiento. Es importante que, el seguimiento al progreso de los alumnos esté combinado de diferentes formas de valoración como: la auto-evaluación, la coevaluación y la heteroevaluación. Lo anterior entendiendo que la evaluación requiere no ser enmarcada en un libreto único, sino que se puede dar de diferentes maneras y en diversos momentos.

\section{Resolución de problemas}

Otra de las alternativas propuestas para el desarrollo adecuado de los procesos evaluativos, en la Institución Educativa Rancho Grande del Municipio de Rondón (Boyacá), es la competencia transversal resolución de problemas, que tiene como objetivo contribuir al desarrollo del pensamiento matemático apoyado en el trabajo grupal e individual.

Según Sánchez (2003),

La resolución de problemas es un medio que promueve el desarrollo continuo del aprendizaje, el cual lo ha convertido en una estrategia indispensable en la formación por el formato autónomo que maneja, de manera que debe ser incluido en los diferentes planes de sesión de clase como valor agregado de los métodos de enseñanza. (p. 87).

El pensamiento matemático en resolución de problemas incluye la actividad cognitiva (por ejemplo, representar el problema, comprenderlo, planificar la resolución, utilizar estrategias) y también actividades que intentan controlar $\mathrm{y}$ regular los propios procesos cognitivos y que, en gran medida, determinan el éxito o el fracaso de la resolución.

Algunos autores agrupan estas actividades bajo etapas o fases, donde cada una es importante y un buen resolutor debería seguirlas. Según Pólya (citado en Castro, 2011), existen cuatro fases: comprensión, planificación, ejecución y revisión.

La competencia resolución de problemas, a partir de la evaluación participativa, le ayuda al alumno a saber si está mejorando su aprendizaje, a ubicar su desempeño respecto de los objetivos y en comparación con sus compañeros, a reconocer la ayuda que necesita y lo que debe hacer, impulsándolo a asumir la responsabilidad de su rendimiento (autoevaluación). A los padres, les permite observar avances en el desempeño pedagógico de sus hijos y reconocer formas en las que pueden apoyarlos para mejorar. En la escuela, la evaluación de aprendizajes en el aula permite identificar los logros en la implementación del currículo y las necesidades de trabajo cooperativo para resolver los problemas señalados $y$, de una u otra forma, contribuye al mejoramiento de la calidad educativa de la institución.

Pólya (1965), a través del libro "How to solve it", introduce el término "heurística" para describir el arte de la resolución de problemas. "La heurística 
trata de comprender el método que conduce a la solución de problemas, en particular las operaciones mentales típicamente útiles en este proceso" (p. 102).

Con relación a los postulados de Pólya, los autores Laya, Fernández y González (2009), sostienen que la heurística tiende a la generalidad, al estudio de los métodos, independientemente de la cuestión tratada y se aplica a problemas de todo tipo; constituye acciones que pueden resultar de utilidad para resolver problemas, para lo que se necesita, primero que todo, comprender el problema con sus datos y condicionamientos, concebir un plan donde se profundice sobre el problema existente, ejecución de ese plan, comprobar cada uno de los pasos planeados y una visión retrospectiva para verificar los resultados obtenidos.

Siguiendo la función resolución de problemas en los procesos evaluativos en la Institución Educativa Rancho Grande, del Municipio de Rondón (Boyacá), se propone que la sustentación del problema planteado esté acompañada de ayudas visuales, como elementos didácticos o materiales gráficos, con el propósito que la trasmisión de las ideas tenga más sentido para el auditorio.

De otro lado, es imprescindible mencionar el proceso diseñado por el Ministerio de Educación Nacional de Colombia (s.f.), en el material Pres -guía de enseñanza para docentes de primaria etapas de la resolución de problemasque inicia con la fase de comprensión, donde se determina la presentación del contexto de la situación problema, señalando que, mediante una lectura acompañada con material de apoyo, se busca que los estudiantes determinen cuál es la tarea a realizar; actividad que finaliza con la realización de un plan de acción mediado por un esquema de solución que el Evaluador tendrá preparado de antemano, pero que construirá en conjunto con sus estudiantes, apoyándose en sus ideas (Ministerio de Educación Nacional, 2015).

Es así como, a través de la competencia resolución de problemas, el pensamiento matemático ayuda a mejorar las respuestas académicas en todas las áreas del conocimiento, por cuanto favorecen los procesos metacognitivos y potencializan las habilidades del pensamiento superior. Se considera de gran importancia, revisar y reflexionar sobre los elementos de evaluación que se llevan a cabo en las aulas de clase, con el fin de romper con el paradigma de una evaluación cuantitativa $y$ sancionatoria, bacia una evaluación de carácter formativa, continua $y$ sistemática que tiene como propósito el desarrollo de la competencia resolución de problemas del área de Matemáticas.

Ahora bien, continuando con el tema resolución de problemas, se aborda la etapa de descontextualización, es aquella en donde se enfocan varios centros de aprendizaje, cada uno con una serie de actividades realizadas por fuera del contexto de la situación problema; es donde los estudiantes construyen y afianzan conceptos, desarrollan procesos, comprenden y practican procedimientos necesarios

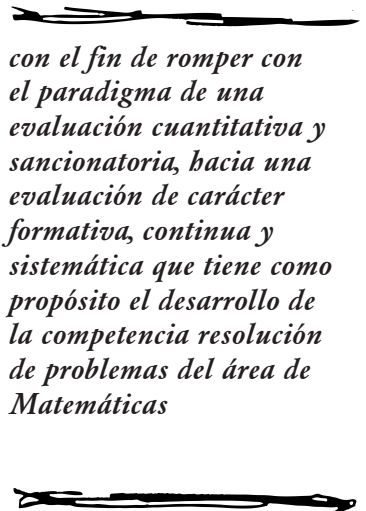


para resolver la situación problema mediante la exploración y construcción de los conceptos grupales, dejando registros escritos de los aprendizajes alcanzados. Seguidamente, se pasa a la etapa de ejercitación y afianzamiento de conceptos y procedimientos (MEN, s.f.). Se finaliza con una situación de aplicación, que le permite al Docente evaluar el aprendizaje de sus estudiantes y su capacidad de transferir lo aprendido a otros contextos.

Es preciso comprender que, la etapa de resolución se inicia con un retorno al esquema de la situación problema realizado en la etapa de comprensión y un enriquecimiento del mismo a partir de los conceptos y procedimientos desarrollados durante los centros de aprendizaje (MEN, s.f.). Cada estudiante diseña una estrategia de resolución para la cual debe definir un orden y una combinación apropiada de los conceptos y procedimientos adquiridos previamente. Finalmente, se comparten y contrastan las diversas estrategias de resolución y se procede a una validación de la solución.

En el mismo sentido, respecto a los formadores Espinoza, Lupiañez y Segovia (2014), establecen que, a partir de la resolución de problemas, "[...] los docentes pueden emplear este tipo de actividades para valorar el grado de comprensión de los conceptos matemáticos aprendidos por los estudiantes, luego de explicar el contenido a enseñar [...]”. (p. 7). También, según coinciden los autores anteriores, esta función puede ser utilizada para hacer un diagnóstico inicial individual y grupal de las capacidades de los educandos, al iniciar el periodo escolar.

En la etapa de reflexión, según el MEN (s.f.), se da el proceso de metacognición que se realiza colectivamente: los estudiantes, guiados por preguntas, reflexionan sobre lo aprendido y sobre su proceso de aprendizaje y toman conciencia de sus procesos mentales. Esta etapa facilita la transferencia de conocimientos en posibles situaciones futuras dentro y fuera del aula.

Posteriormente, se desarrolla la etapa de memorias colectivas, donde los estudiantes generan diferentes estrategias, propuestas, modelos y demás elementos relacionados, directa e indirectamente, con la situación problema. Incluye, entre otros, una cartelera con estrategias de comprensión de la situación problema y de la tarea a realizar; una, con estrategias de solución; otra, con conceptos y procedimientos matemáticos, y otra, de resumen de los aprendizajes alcanzados a lo largo de la secuencia. Las memorias colectivas tienen como propósito: documentar el proceso de resolución de la situación problema, apoyar los distintos momentos del aprendizaje y, como su nombre lo indica, dejar una memoria de los aprendizajes logrados por la clase, que sirve de apoyo para actividades futuras a lo largo del año académico.

Por su parte, Martínez y Ramírez (2016), en cuanto a la competencia de resolución de problemas, resaltan la importancia del trabajo colaborativo en el proceso de enseñanza aprendizaje, gracias a la 
interacción que exige su desarrollo, que puede ser bien complementado con herramientas innovadoras y actualizadas como las Tic y sus diferentes aplicaciones, estrategias que llaman la atención de los alumnos porque rompen con la tradicionalidad de las actividades en el aula de clase.

En el proceso planteado por el Ministerio de Educación Nacional de Colombia, en el material de la Resolución de Problemas, la labor del docente está enfocada en varios aspectos. Inicialmente, él debe fomentar, en los estudiantes, actitudes positivas y aprecio hacia las matemáticas; ayudarlos a desarrollar seguridad y confianza en sí mismos, induciéndolos a desarrollar el interés en hacer preguntas, expresar ideas propias y solicitar justificaciones o explicaciones para cualquier respuesta o procedimiento suministrado por otra persona; con lo anterior, se busca profundizar en el conocimiento y comprensión de los estudiantes, seguridad a la hora de hacer conjeturas y evaluarlas, preguntar por qué, explicar su razonamiento y argumentar; igualmente, perseverancia en el proceso de aprendizaje, iniciativa para intentar diversas estrategias, la convicción de la utilidad de las matemáticas y el poder de sus argumentos; el interés por su aprendizaje y la valoración de su belleza, y visión del error como una oportunidad para aprender.

Finalmente, es importante enfocar este análisis en la evaluación formativa con el fin de acompañar y apoyar a cada estudiante en su proceso de aprendizaje, es necesario evaluar si está alcanzando los aprendizajes esperados durante cada una de las etapas de la secuencia.

Picaroni (2011) define evaluación formativa como:

Aquella que posibilita un doble aprendizaje. Por un lado, indica al estudiante en qué parte del proceso se encuentra, respecto a las diferentes fases por las que puede pasar en su desarrollo; por otro, informa al docente sobre los efectos de su labor de enseñanza, indicando los logros y las dificultades del que aprende. El concepto de evaluación formativa remite a una caracterización dinámica de la situación educativa, en la que ocurren interacciones entre los elementos que la configuran: alumnos, docente, conocimientos. (p. 15).

En apoyo de los anteriores referentes, es oportuno indicar que, en la Institución Educativa Rancho Grande, del Municipio de Rondón (Boyacá), la evaluación formativa tendría como finalidad generar la información numérica y cualitativa, sobre los logros y falencias de cada educando, sin establecer conceptos de juicio, por el contrario, transmitiendo mensajes de motivación para mejorar.Para robustecer la apreciación anterior, es oportuno citar a Pinilla (2013), quien denomina la evaluación formativa como:

El examen de desempeño de un estudiante en relación con sus objetivos cognitivos, en modo de favorecerla sobre la base de los resultados; se incluye en esta, por lo
El examen de desempeño de un estudiante en relación con sus objetivos cognitivos, en modo de favorecerla sobre la base de los resultados 
general, la evaluación diagnóstica en la cual se identifican las dificultades del estudiante, sea en lo relacionado con el aprendizaje, sea por lo que respecta la falta de comprensión. (p. 48).

En el epílogo de esta reflexión, se concibe que en la Institución Educativa Rancho Grande del Municipio de Rondón (Boyacá), el proceso de evaluación formativa debe infundir el deseo de búsqueda permanente del crecimiento en el conocimiento individual y colectivo de los estudiantes, que induzcan al constante desarrollo creativo e innovador, con base en los métodos efectivos y técnicas innovadoras de la enseñanza y el aprendizaje.

\section{Conclusiones}

Los docentes consideran estar muy bien en prácticas docentes, sin embargo, se nota debilidades en el uso de material concreto, uso de referentes de calidad, planeación estructurada en sus diferentes momentos, uso efectivo del tiempo, procesos de evaluación, trabajo colaborativo y hasta manejo disciplinar del área entre otros factores.

Los docentes del área opinan en general que, en el centro de la educación es de menos importancia la evaluación sumativa, frente a la formativa, aspecto que en la realidad no se aprecia, teniendo en cuenta que los alumnos reciben evaluaciones calificadas y no cualificadas, es decir, el juicio de valor es numérico y algunas veces incluye solo comentarios cortos sobre la necesidad de aumentar el esfuerzo o mejoramiento en el aprendizaje.
De esta forma, la filosofía al respecto de la Institución, debe basarse en la nueva cultura evaluativa que busque resaltar el papel que representa la evaluación en los procesos de enseñanza-aprendizaje. De manera que, la diferencia entre el control sumativo y el formativo de la evaluación, es la valoración del aprendizaje en la primera; y la evaluación para el aprendizaje, en la segunda.

Se estableció que hay docentes que solo basan el logro de resultados en el mecanismo de los instrumentos que generan cifras cuantitativas, para mostrar el nivel de aprendizaje de los educandos, dejando de lado el verdadero potencial formativo que puede aportar una estrategia evaluativa con fondo analítico y organizado, para implementar pedagogías que favorezcan el interés de los alumnos por aprender; de esta forma, las herramientas evaluativas no solo serán registros que proporcionan resultados.

Los docentes en general tienen la preocupación constante de que sus estudiantes desarrollen una mejora continua en el aprendizaje, pero es una constante que no todos los profesores centran el análisis de los resultados del proceso de enseñanza-aprendizaje, en la identificación de las verdaderas necesidades cognitivas de los alumnos.

Se busca diseñar una estrategia metodológica que oriente a los docentes en la implementación de la evaluación formativa articulada o aplicada desde la resolución de problemas, propone trabajar la evaluación formativa articulada en tres fases de la resolución 
de problemas propuesto por el MEN en el material PREST (comprensión, resolución y reflexión, evidenciado en cada fase de la resolución de problemas los diferentes momentos la evaluación (autoevaluación, coevaluación y heteroevaluación) y los elementos más importantes que se deben tener en cuenta para valorar los aprendizajes de los estudiantes, como son el identificar las tareas a desarrollar, esquematizar la situación problema, socializar con los compañeros de la mesa de trabajo y con todos los asistentes en el salón, estrategias de solución, validación frente a otros equipos de trabajo y participación en la creación de memorias colectivas. Este documento queda a disposición de los docentes como aporte al proceso de evaluación, que contribuya a una educación de calidad.

Finalmente, se concibe por parte de los docentes que, aún falta aproximar los diseños metodológicos de los procesos evaluativos a los verdaderos fines de estos. Lo que hace que la propuesta aquí presentada, responda de manera clara a las necesidades del contexto educativo de la Institución.

\section{Referencias}

Aldana, E., \& Wagner, G. (2012). La evaluación del aprendizaje en matemáticas. Medellín: Sello Editorial Universidad de Medellín.

Castro, E. (2011). La invención de problemas y sus ámbitos de investigación. En J. Lupiáñez et al. (eds.), Investigaciones en pensamiento numérico y algebraico e bistoria de la matemática y educación matemática (pp. 1-15). Granada: Universidad de Granada.

Cardona, S. A., Jaramillo, S., \& Navarro, Y. (2016). Evaluación de competencias con apoyo de un sistema de gestión de aprendizaje. Praxis \& Saber, 7(14), 193-218. https://doi.org/10.19053/22160159.5223

Espinoza, J., Lupiañez, J. L., \& Segovia, I. (2014). La invención de problemas y sus ámbitos de investigación en educación matemática. Revista Digital: Matemática, Educación e Internet, 14(2), 1-12. DOI: http://dx.doi.org/10.18845/ rdmei.v14i2.1664.

Flores M. \& González, O. (1999). El trabajo docente: enfoques innovadores para el diseño de un curso. México: Trillas.

Gómez, R., \& Roquet, V. (2012). Metodología de la investigación. México: Red Tercer Milenio. Recuperado de http://www.aliatuniversidades.com.Mx/ bibliotecasdigitales/pdf/ Axiológicas/Metodología_de_la_Investigación.pdf. 
Laya, M., Fernández, R., \& González, S. (2009). Método y estrategias de resolución de problemas matemáticos utilizadas por alumnos de 6to. Grado de primaria. México: Universidad Iberoamericana.

Martínez, P., \& Ramírez, A. (2016). Diseño y aplicación de una estrategia de gestión educativa para fortalecer la competencia resolución de problemas matemáticos en el grado 5 del Colegio Villa Rica (IED). (Tesis de Maestría). Universidad Libre de Colombia, Bogotá, Colombia.

Moreno, I., \& Ortiz, J. (2016). Docentes de educación básica y sus concepciones acerca de la evaluación en matemática. Revista Iberoamericana de Evaluación Educativa, 1(1), 140-154.

Ministerio de Educación Nacional . (2009). Fundamentaciones y orientaciones para la implementación del Decreto 1290 de 2009. Obtenido de mineducacion.gov. co: https://www.mineducacion.gov.co/1621/articles-213769_archivo_pdf_ evaluacion.pdf Picaroni, B. (2011). La evaluación en las aulas de primaria: usos formativos, calificaciones y comunicación con los padres.

Pinilla, M. (2013). Múltiples aspectos del aprendizaje de la matemática. Bogotá: Editorial Magisterio.

Pólya, G. (1965). Cómo plantear y resolver problemas. México: Trillas.

Salkind, N. (1999). Métodos de investigación. México: Prentice-Hall (3a ed.). .Sánchez, N. M. (2003). La resolución de problemas matemáticos. Una caracterización histórica de su aplicación como vía eficaz para la enseñanza de la matemática. Pedagogía Universitaria, 8(3), 81-88.

Villa, A \& Henao, B. (2009) Aportes a la discusión sobre los procesos metodológicos y evaluativos en el ámbito de la formación de maestros de la Facultad de Educación de la universidad de Antioquia. Unipluridiversidad. 